\title{
Diffraction efficiency and aberrations of diffractive elements obtained from orthogonal expansion of the point spread function \\ Jim Schwiegerling*a \\ ${ }^{a}$ College of Optical Sciences, University of Arizona, Tucson, AZ 85721
}

\begin{abstract}
The Point Spread Function (PSF) indirectly encodes the wavefront aberrations of an optical system and therefore is a metric of the system performance. Analysis of the PSF properties is useful in the case of diffractive optics where the wavefront emerging from the exit pupil is not necessarily continuous and consequently not well represented by traditional wavefront error descriptors such as Zernike polynomials. The discontinuities in the wavefront from diffractive optics occur in cases where step heights in the element are not multiples of the illumination wavelength. Examples include binary or N-step structures, multifocal elements where two or more foci are intentionally created or cases where other wavelengths besides the design wavelength are used. Here, a technique for expanding the electric field amplitude of the PSF into a series of orthogonal functions is explored. The expansion coefficients provide insight into the diffraction efficiency and aberration content of diffractive optical elements. Furthermore, this technique is more broadly applicable to elements with a finite number of diffractive zones, as well as decentered patterns.
\end{abstract}

Keywords: Diffractive lenses, diffraction efficiency, point spread function, orthogonal expansion

\section{INTRODUCTION}

Diffractive lenses have evolved in their complexity over the past few decades. Lithographic techniques provided for binary or N-step structures. Technologies such has single-point diamond turning and glass and plastic molding processes have facilitated the implementation of kinoform lenses, in which the discrete diffractive steps of the lens are separated by a smoothly varying region. Diffractive lenses are increasingly being used in commercially available lenses. For example, Canon has a line of DSLR lenses that incorporate a multi-layer diffractive element.[1] Such a structure provides the lens designer with additional capabilities in terms of aberration and color correction. Diffractive lenses have also found wide commercial application in ophthalmic optics. Bifocal[2] and more recently trifocal[3] intraocular lenses have found success in treating presbyopic patients by giving them multiple discrete planes of sharp focus. In evaluating the performance of diffractive lenses, the diffraction efficiency is a key metric. This number describes the fraction of energy going into each of the diffractive orders. In the case of the DSLR lenses, the ideal diffractive structure would have a diffraction efficiency of unity for a single diffractive order, and consequently zero efficiency for all other diffractive orders. For the bifocal and trifocal lenses, the diffraction efficiency is ideally split between two and three orders, respectively. Theoretical and fabrication limitations do not allow these ideal diffractive structures to be realized. Understanding the leakage of energy into non-desired diffraction orders defines the performance limits of such systems. Here, a generalized expression for the diffraction efficiency applicable to a broad range of diffractive elements is developed. The derivation involves a decomposition of the Point Spread Function (PSF) and analysis of the resulting expansion coefficients.

\section{PSF EXPANSION}

In general, the complex pupil function in terms of Zernike polynomials $Z_{n}^{m}(\rho, \theta)$ is given by

$$
P(\rho, \theta)=\operatorname{cyl}\left(\frac{\rho}{2}\right) \exp \left[-\sum_{n^{\prime}, m^{\prime}} c_{n^{\prime} m^{\prime}} Z_{n^{\prime}}^{m^{\prime}}(\rho, \theta)\right]
$$

where

$$
c_{n^{\prime} m^{\prime}}=\alpha_{n^{\prime} m^{\prime}}+\frac{i 2 \pi}{\lambda} a_{n^{\prime} m^{\prime}}\left(h_{x}, h_{y}\right) .
$$

*jschwieg@u.arizona.edu; phone+1(520)621-8688

Optical Modeling and Performance Predictions VIII, edited by Mark A. Kahan,

Marie B. Levine-West, Proc. of SPIE Vol. 9953, 995307 - @ 2016 SPIE

CCC code: $0277-786 X / 16 / \$ 18 \cdot$ doi: $10.1117 / 12.2237907$ 
The coefficients $\alpha_{n^{\prime} m^{\prime}}$ describe the pupil apodization, and the coefficients $a_{n^{\prime} m^{\prime}}\left(h_{x}, h_{y}\right)$ describe the wavefront error. In general, these latter coefficients are functions of the field coordinates $\left(h_{x}, h_{y}\right)$. The entire complex exponential of the pupil function can be decomposed into Zernike polynomials such that

where

$$
P(\rho, \theta)=\operatorname{cyl}\left(\frac{\rho}{2}\right) \sum_{n m} b_{n m} \tilde{Z}_{n}^{m}(\rho, \theta)
$$

$$
b_{n m}=\frac{n+1}{\pi} \int_{0}^{2 \pi} \int_{0}^{1} \exp \left[-\sum_{n^{\prime}, m^{\prime}} c_{n^{\prime} m^{\prime}} Z_{n^{\prime}}^{m^{\prime}}(\rho, \theta)\right] \tilde{Z}_{n}^{m}(\rho, \theta) \rho d \rho d \theta
$$

and $\tilde{Z}_{n}^{m}(\rho, \theta)=R_{n}^{m}(\rho) \exp (\operatorname{im} \theta)$ are the complex version of the Zernike polynomials with $R_{n}^{m}(\rho)$ being the Zernike radial polynomials. The coefficients $b_{n m}$ will depend on the field coordinates, but the field coordinates are simply suppressed for compactness. These expansion coefficients play an essential role in the description of the diffraction efficiency.

The amplitude point spread function $a P S F$ is the Fourier transform of the pupil function

$$
\operatorname{aPSF}(r, \varphi)=\int_{0}^{2 \pi} \int_{0}^{\infty} P(\rho, \theta) \exp [-i 2 \pi \rho \cos (\theta-\varphi)] \rho d \rho d \theta
$$

where the Fourier transform has been converted to polar coordinates. Inserting the pupil function in Eq. 5 and using the Bessel function relationships $J_{m}(-x)=(-1)^{m} J_{m}(x)$ and

$$
\int_{0}^{2 \pi} \exp (i m \alpha) \exp (i x \cos \alpha) d \alpha=2 \pi i^{m} J_{m}(x)
$$

gives

From Janssen[4]

$$
\operatorname{aPSF}(r, \varphi)=2 \pi \sum_{n, m} b_{n m}(-i)^{m} \exp (i m \varphi) \int_{0}^{1} R_{n}^{m}(\rho) J_{m}(2 \pi \rho r) \rho d \rho
$$

so

$$
\int_{0}^{1} R_{n}^{m}(\rho) J_{m}(2 \pi \rho r) \rho d \rho=(-1)^{(n-m) / 2} \frac{J_{n+1}(2 \pi r)}{2 \pi r},
$$

$$
\operatorname{aPSF}(r, \varphi)=\sum_{n, m} b_{n m}(-i)^{n} \exp (i m \varphi) \frac{J_{n+1}(2 \pi r)}{r} .
$$

The intensity Point Spread Function (PSF) is the squared modulus of Eq. 9 given by

$$
\operatorname{PSF}(r, \varphi)=\left(\sum_{n, m} b_{n m}(-i)^{n} \exp (i m \varphi) \frac{J_{n+1}(2 \pi r)}{r}\right) \times\left(\sum_{n^{\prime} m^{\prime}} b_{n^{\prime} m^{\prime}}^{*}(i)^{n^{\prime}} \exp \left(-i m^{\prime} \varphi\right) \frac{J_{n^{\prime}+1}(2 \pi r)}{r}\right) .
$$

In multiplying these two series, the initial term has a coefficient $\left|b_{00}\right|^{2}$ and its functional form is simply the Airy pattern. The additional terms in Eq. 10 describe both broader rotationally symmetric terms, as well as non-rotationally symmetric components of the PSF. In the normalization scheme used here, this coefficient $\left|b_{00}\right|^{2}$ is a number between zero and one that describes the amount of energy contained with Airy pattern term. It is this value, at least for on-axis rotationally symmetric system, that will be used as the definition of the diffraction efficiency. 


\section{GENERALIZED DIFFRACTION EFFICIENCY}

A wide range of rotationally symmetric diffractive phase profiles can be described by the general parabolic piecewise formula

$$
\phi_{\text {diff }}\left(\rho^{2}\right)=2 \pi\left[\alpha_{j} \frac{\left(r_{\max } \rho^{2}-r_{j-1}^{2}\right)}{\left(r_{j}^{2}-r_{j-1}^{2}\right)}+\beta_{j}\right] \quad \text { for } \frac{r_{j-1}^{2}}{r_{\max }^{2}} \leq \rho^{2}<\frac{r_{j}^{2}}{r_{\max }^{2}} \text { and } j=0,1,2 \ldots J .
$$

where $r_{\max }$ is the maximum radial coordinate $r_{j}$ are the locations of the boundaries of annular regions within the pupil. The constants $\alpha_{j}$ and $\beta_{j}$ describe the phase profile over the $j t h$ annular ring. For convenience, $r_{-1}$ is defined as zero. Eq. 11 is versatile. Setting all the $\alpha_{j}$ to zero enables the definition of binary or $\mathrm{N}$-step phase profiles, whereas non-zero values for these constants enable a parabolic arc and a constant offset to be introduced for each annular zone. The coefficients $\alpha_{j}$ and $\beta_{j}$ have a simple interpretation. At the inner edge of a diffractive zone (i.e. $\rho=\rho_{\mathrm{j}-1}$ ), the relative phase is $\beta_{\mathrm{j}}$. At the outer edge of the diffractive zone (i.e. $\rho=\rho_{\mathrm{j}}$ ), the relative phase is $\alpha_{\mathrm{j}}+\beta_{\mathrm{j}}$. Note also at this point, no relationship between the annular zone boundaries has been assumed.

From Eq. 4

$$
b_{00}=2 \int_{0}^{1} P(\rho) \exp \left[i 2 \pi W_{20} \rho^{2}\right] \rho d \rho,
$$

where $W_{20}$ is the defocus coefficient in waves. The pupil function in the $j t h$ annular zone is given by $P(\rho)=$ $\exp \left[-i \phi_{\text {diff }}\left(\rho^{2}\right)\right]$. Each of the annular zones can be integrated separately and summed to solve Eq. 12, leading to

$$
b_{00}=\sum_{j=0}^{J}\left(\rho_{j}^{2}-\rho_{j-1}^{2}\right) \exp \left[-i 2 \pi\left(\beta_{j}+\frac{\alpha_{j}}{2}-\frac{W_{20}}{2}\left(\rho_{j}^{2}+\rho_{j-1}^{2}\right)\right)\right] \operatorname{sinc}\left[W_{20}\left(\rho_{j}^{2}-\rho_{j-1}^{2}\right)-\alpha_{j}\right],
$$

where the $\rho_{\mathrm{j}}$ are just the normalized coordinate versions of the annular ring boundaries $r_{j}$. Eq. 13 is general and only assumes a rotationally symmetric diffractive phase profile described by the piecewise quadratic phase profile of Eq. 11 . The squared modulus of this expression $\left|b_{00}\right|^{2}$ for specific levels of defocus will be used as the diffraction efficiency value.

\section{USEFUL MATH RELATIONSHIPS}

There are several of mathematical definitions and derivations that will be useful for subsequent derivations of the diffraction efficiency. First, define a variation on the Kronecker delta function, which will be referred to as the Kronecker odd delta as

$$
\delta_{\text {odd } j}=\left\{\begin{array}{ll}
1 & \text { for } j \text { odd } \\
0 & \text { for } j \text { even }
\end{array} .\right.
$$

Second, define the $\operatorname{sinj}($ ) function (pronounced "Singe”) as

$$
\operatorname{sinj}(J ; x):=\frac{\operatorname{sinc}(J x)}{\operatorname{sinc}(x)}
$$

where $J$ is an integer. When $J$ is an even integer, the $\operatorname{sinj}($ ) function alternates between \pm 1 when $x$ is an integer. When $J$ is an odd integer, the $\operatorname{sinj}($ ) function is unity when $x$ is an integer. For non-integer values of $x$, the $\operatorname{sinj}($ ) function tends towards zero, especially as $J$ becomes large. Figure 1 shows examples of the $\sin j($ ) function for $J=16$ and $J=17$.

Finally, the mathematical result based on the properties of a geometric series

$$
\sum_{k=0}^{n} \exp (i 2 \alpha \pi)^{k}=(n+1) \exp (i \alpha n \pi) \operatorname{sinj}(n+1 ; \alpha)
$$

will prove useful as well. 

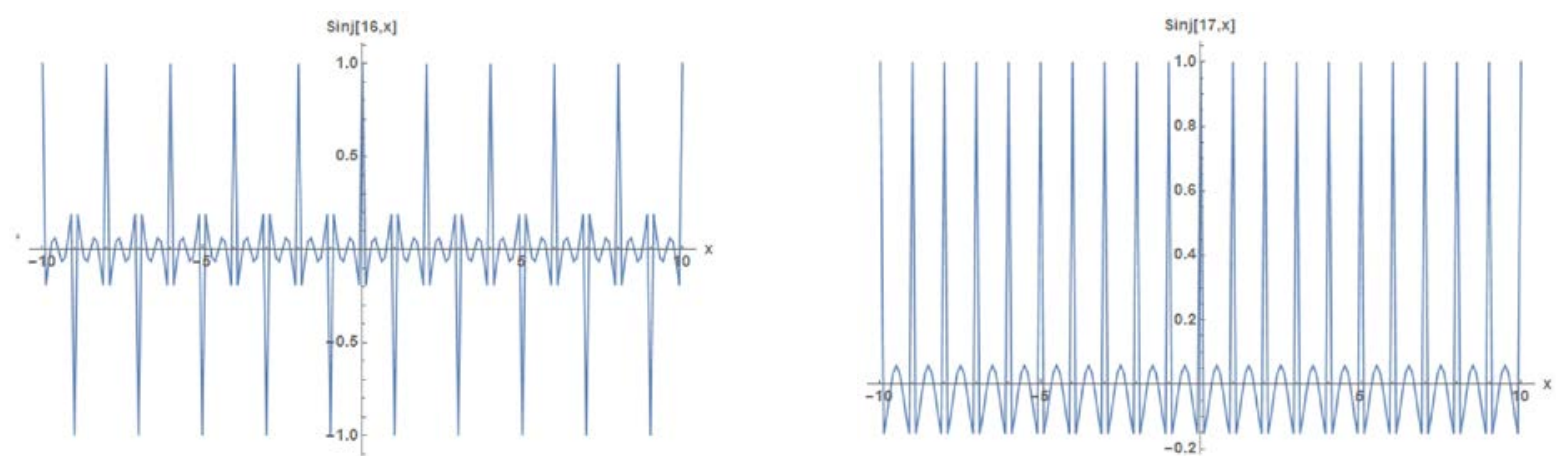

Figure 1. Examples of the $\operatorname{sinj}$ ( ) function for $J=16$ (left) and $J=17$ (right).

\section{EXAMPLE CALCULATIONS}

For diffractive lenses, the lens aperture is typically broken up into annular regions of equal area. Occasionally, the central circular region of the lens will have a different area than the remaining diffractive zones, but for simplicity here, only equiarea zones will be considered. Under this assumption, the locations of the boundaries of the transition zones are given by

$$
r_{j}=\sqrt{\frac{2 j \lambda_{o}}{\phi_{a d d}}},
$$

where $\lambda_{o}$ is the design wavelength and $\phi_{a d d}$ is the power of the diffractive lens. The positions of the transitions in normalized coordinates is simply given by $\rho_{j}=r_{j} / r_{\max }$.

It is also useful to convert the defocus coefficient $W_{20}$ into an expression proportional to power $\phi_{d e f}$. To do so, define

$$
W_{20}=\frac{\phi_{\text {def }} r_{\max }^{2}}{2 \lambda_{o}}
$$

\subsection{Bifocal Diffractive Lens}

Utilizing the framework defined above, a standard bifocal diffractive lens is defined with $\alpha_{j}=$ constant $:=\alpha_{1}$ and $\beta_{j}=0$. Figure 2 shows the phase profile for the case where $\phi_{a d d}=4$ diopters and $\alpha_{1}=0.5$ (i.e. a diffractive step height of half the design wavelength).

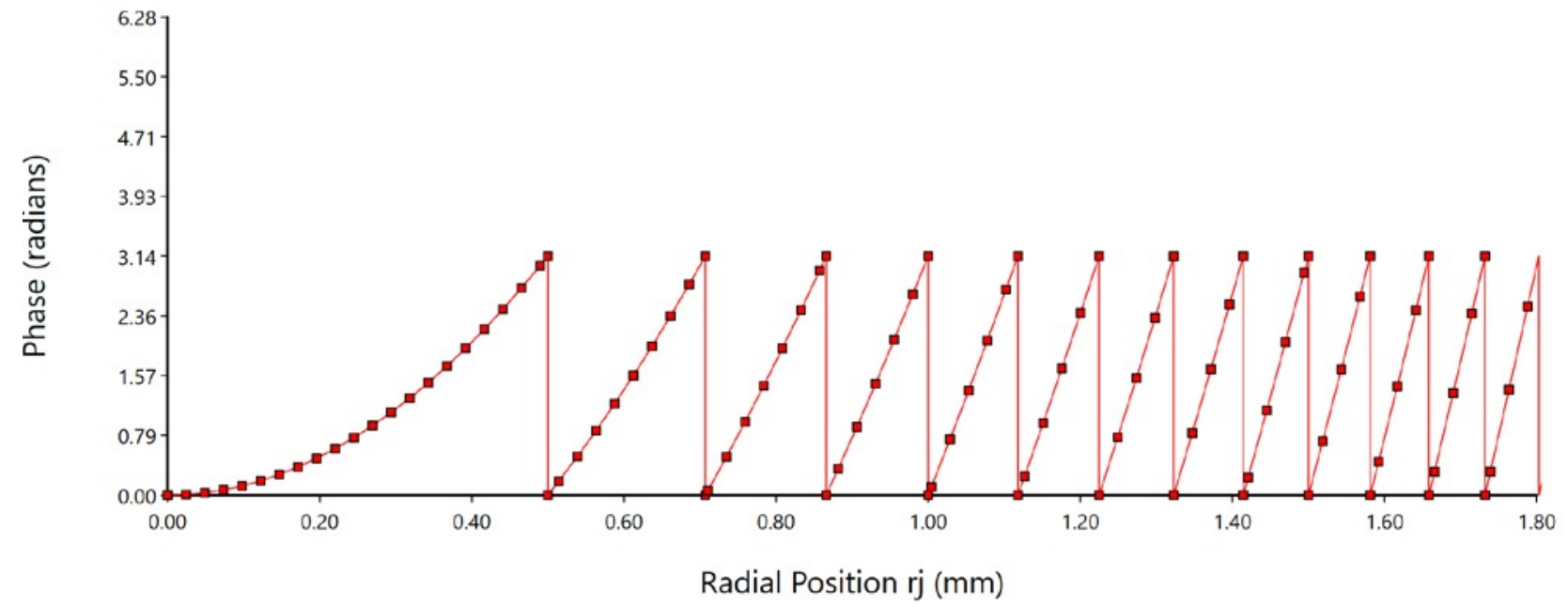

Figure 2. Phase profile for a 4.00 diopter bifocal diffractive lens with $\alpha_{1}=0.5$ (half-wave or $\pi$ radians). 
Since the diffractive lens consists of $J$ diffractive zones of equal area, it is easy to see that

Using this result in Eq. 13 gives

$$
\rho_{j}^{2}-\rho_{j-1}^{2}=\frac{1}{J}
$$

$$
b_{00}=\sum_{j=1}^{J} \frac{1}{J} \exp \left[-i 2 \pi\left(\frac{\alpha_{1}}{2}-\left(j-\frac{1}{2}\right) \frac{\phi_{\text {def }}}{\phi_{\text {add }}}\right)\right] \operatorname{sinc}\left[\frac{\phi_{\text {def }}}{\phi_{\text {add }}}-\alpha_{1}\right] .
$$

Factoring out all of the terms independent of $j$ and using the result of Eq. 16 gives a diffraction efficiency of

$$
\left|b_{00}\right|^{2}=\operatorname{sinc}^{2}\left[\frac{\phi_{\text {def }}}{\phi_{\text {add }}}-\alpha_{1}\right] \operatorname{sinj}^{2}\left[J ; \frac{\phi_{\text {def }}}{\phi_{\text {add }}}\right] \text {. }
$$

Figure 3 shows the diffraction efficiency as $\phi_{d e f}$ is varied for $\phi_{\text {add }}=4.0$ diopters, $\alpha_{1}=0.5$ and $J=24$. The diffraction efficiency is 0.405 when $\phi_{\text {def }}=0$ and $\phi_{\text {def }}=\phi_{\text {add }}$. Effectively, these are the $0^{\text {th }}$ and $+1^{\text {st }}$ diffractive orders. Similarly, the diffraction efficiency is 0.045 when $\phi_{d e f}=-\phi_{a d d}$ and $\phi_{d e f}=2 \phi_{a d d}$. These correspond to the $-1^{\text {st }}$ and $+2^{\text {nd }}$ diffractive orders, respectively.

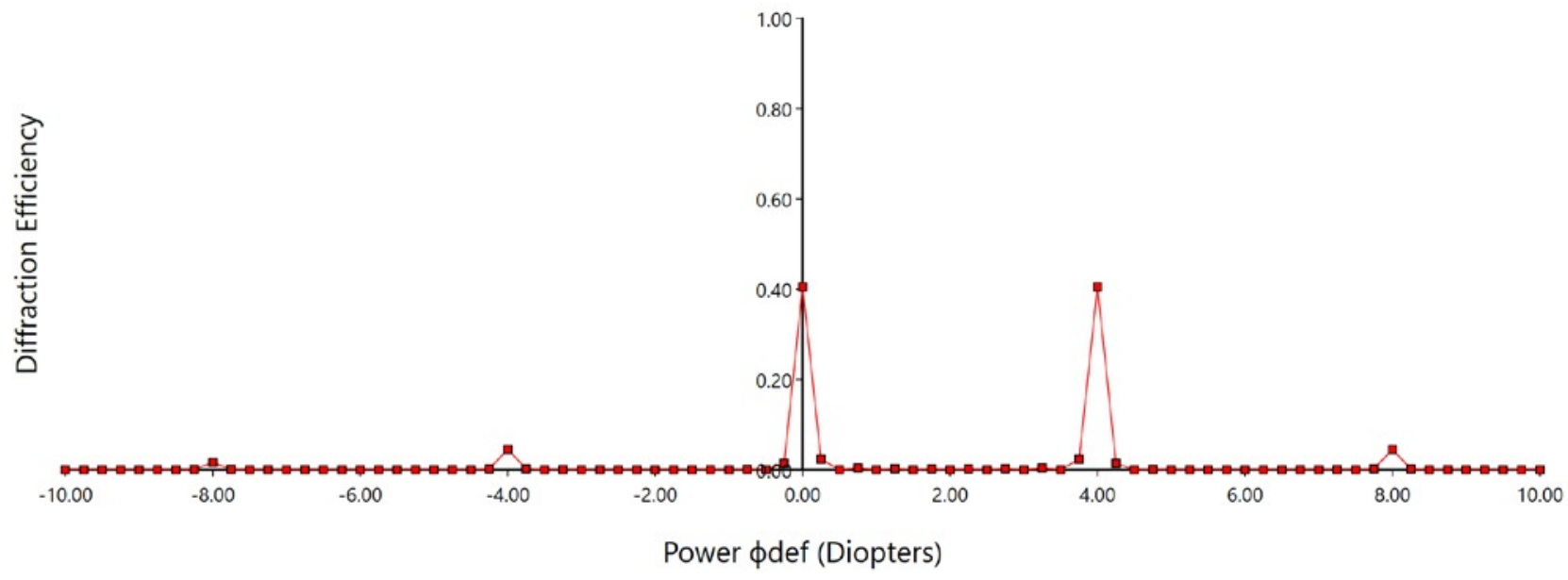

Figure 3. Diffraction efficiency for a 4.00 diopter bifocal diffractive lens with $\alpha_{1}=0.5$ (half-wave or $\pi$ radians) and $J=24$.

\subsection{Trifocal Diffractive Lens}

Next, consider a trifocal diffractive lens with parameters defined with $\alpha_{j}=\left\{\alpha_{1}, \alpha_{2}\right\}$ and $\beta_{j}=\left\{\beta_{1}, \beta_{2}\right\}$, where the diffractive zones alternate between the two values. Again, assuming equal area annular zones, with the zone boundaries defined by Eq. 17 and modifying Eq. 13, the first PSF expansion coefficient is defined as

$$
\begin{aligned}
& \sum_{\substack{j=1 \\
j \text { odd }}}^{\substack{b_{00}=\\
\text { Jodd }}}\left(\rho_{j}^{2}-\rho_{j-1}^{2}\right) \exp \left[-i 2 \pi\left(\beta_{1}+\frac{\alpha_{1}}{2}-\frac{W_{20}}{2}\left(\rho_{j}^{2}+\rho_{j-1}^{2}\right)\right)\right] \operatorname{sinc}\left[W_{20}\left(\rho_{j}^{2}-\rho_{j-1}^{2}\right)-\alpha_{1}\right]+ \\
& \quad \sum_{\substack{j=2 \\
j \text { even }}}^{J_{\text {even }}}\left(\rho_{j}^{2}-\rho_{j-1}^{2}\right) \exp \left[-i 2 \pi\left(\beta_{2}+\frac{\alpha_{2}}{2}-\frac{W_{20}}{2}\left(\rho_{j}^{2}+\rho_{j-1}^{2}\right)\right)\right] \operatorname{sinc}\left[W_{20}\left(\rho_{j}^{2}-\rho_{j-1}^{2}\right)-\alpha_{2}\right],
\end{aligned}
$$


where $J_{\text {odd }}=J$ and $J_{\text {even }}=J-1$ when $J$ is odd. Similarly, $J_{\text {odd }}=J-1$ and $J_{\text {even }}=J$ when $J$ is even. Applying similar techniques to section 5.1 and the results of section 4, the diffraction efficiency for a trifocal diffractive lens as defined above is given by

$$
\begin{gathered}
\left|b_{00}\right|^{2}=\frac{1}{4}\left(\frac{J+\delta_{o d d J}}{J}\right) \operatorname{sinc}^{2}\left[\frac{\phi_{\text {def }}}{\phi_{\text {add }}}-\alpha_{1}\right] \operatorname{sinj}^{2}\left[\frac{J+\delta_{\text {oddJ }}}{2} ; \frac{2 \phi_{\text {def }}}{\phi_{\text {add }}}\right] \\
+\frac{1}{4}\left(\frac{J-\delta_{\text {odd } J}}{J}\right) \operatorname{sinc}^{2}\left[\frac{\phi_{\text {def }}}{\phi_{\text {add }}}-\alpha_{2}\right] \operatorname{sinj}^{2}\left[\frac{J-\delta_{\text {odd }}}{2} ; \frac{2 \phi_{\text {def }}}{\phi_{\text {add }}}\right] \\
+\frac{1}{2}\left(\frac{J^{2}-\delta_{\text {odd J }}}{J^{2}}\right) \operatorname{sinc}\left[\frac{\phi_{\text {def }}}{\phi_{a d d}}-\alpha_{1}\right] \operatorname{sinc}\left[\frac{\phi_{\text {def }}}{\phi_{\text {add }}}-\alpha_{2}\right] \times \\
\operatorname{sinj}\left[\frac{J+\delta_{\text {oddJ }}}{2} ; \frac{2 \phi_{\text {def }}}{\phi_{\text {add }}}\right] \operatorname{sinj}\left[\frac{J-\delta_{\text {oddJ }}}{2} ; \frac{2 \phi_{\text {def }}}{\phi_{\text {add }}}\right] \cos \left[2 \pi\left(\beta_{1}-\beta_{2}+\frac{\alpha_{1}-\alpha_{2}}{2}+\left(1-\delta_{\text {oddJ }}\right) \frac{\phi_{\text {def }}}{\phi_{\text {add }}}\right)\right] .
\end{gathered}
$$

Figure 4 shows the phase profile for the case where $\phi_{\text {add }}=4$ diopters, the steps alternate between $\alpha_{1}=0.311$ waves and $\alpha_{2}=0.689$ waves, and the parameters $\beta_{1}=\beta_{2}=0$.

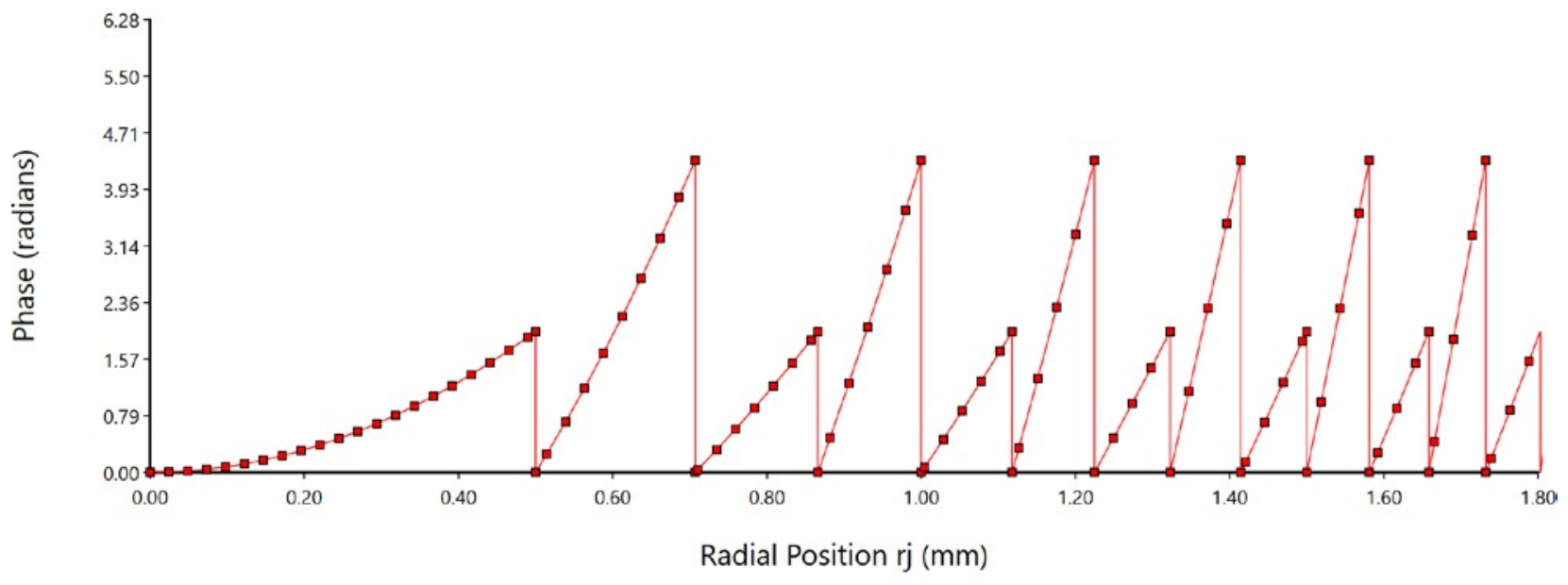

Figure 4. Phase profile for a 4.00 diopter trifocal diffractive lens with diffractive zones alternating between $\alpha_{1}=0.311$ waves (1.954 radians) and $\alpha_{2}=0.689$ waves ( 4.329 radians).

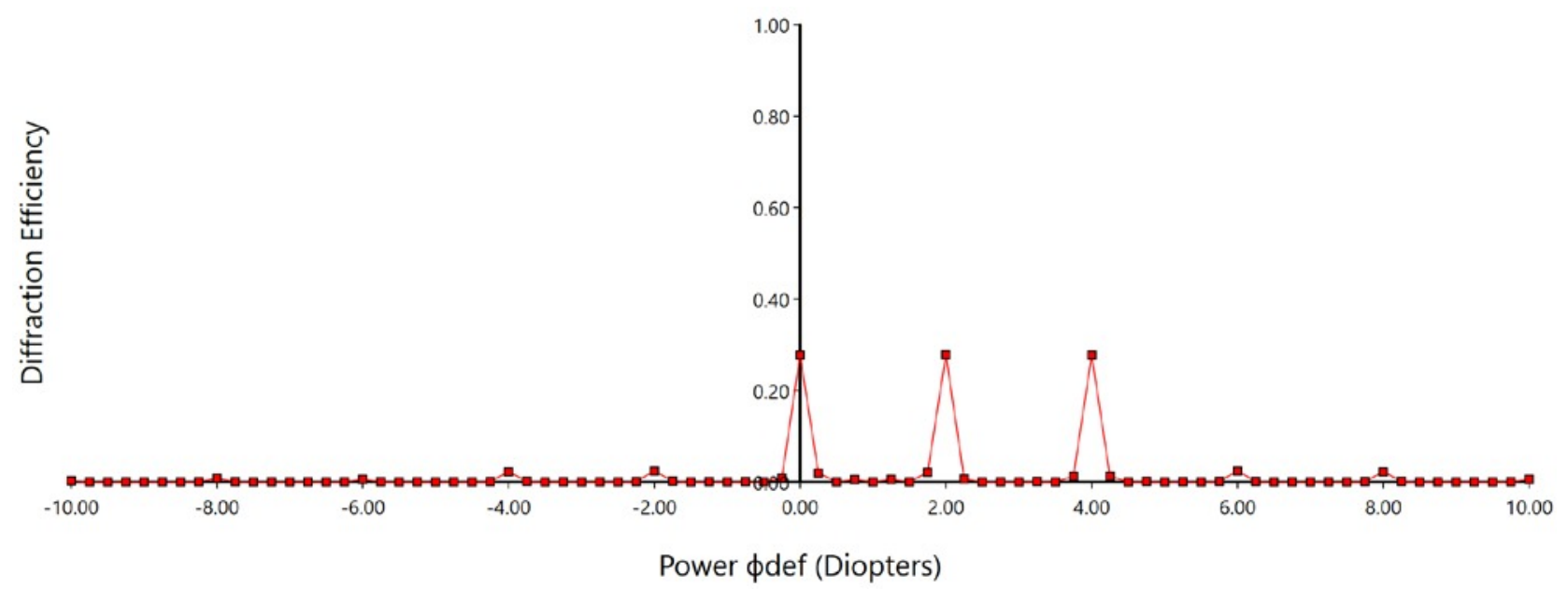

Figure 5. Diffraction efficiency for a 4.00 diopter trifocal diffractive lens with $\alpha_{1}=0.311$ waves (1.954 radians) and $\alpha_{2}=$ 0.689 waves (4.329 radians) and $J=24$. 
Figure 5 shows the diffraction efficiency as $\phi_{d e f}$ is varied for $\phi_{\text {add }}=4.0$ diopters, the diffractive steps alternating between $\alpha_{1}=0.311$ and $\alpha_{2}=0.689$, with $\beta_{1}=\beta_{2}=0$ and $J=24$. The diffraction efficiency is 0.277 when $\phi_{d e f}=0$ and $\phi_{d e f}=$ $0.5 \phi_{\text {add }}$ and $\phi_{\text {def }}=\phi_{\text {add }}$. Effectively, these are the $0^{\text {th }},+1^{\text {st }}$ and $+2^{\text {nd }}$ diffractive orders. Similarly, the diffraction efficiency is 0.024 when $\phi_{d e f}=-0.5 \phi_{a d d}$ and $\phi_{d e f}=1.5 \phi_{a d d}$. These correspond to the $-1^{\text {st }}$ and $+3^{\text {rd }}$ diffractive orders, respectively. Beyond that, the diffraction efficiency is 0.022 when $\phi_{d e f}=-\phi_{a d d}$ and $\phi_{d e f}=2 \phi_{a d d}$. These correspond to the $-2^{\text {nd }}$ and $+4^{\text {th }}$ diffractive orders, respectively.

\section{SUMMARY}

Diffraction efficiency is a measure of the performance of diffractive lenses. Here a generalized definition of diffraction efficiency is developed based on a linear expansion of the PSF into a set of basis functions. For the rotationally symmetric case, the on-axis performance describes the diffraction efficiency. The expression given in Eq. 13 is general in the sense that it only assumes a circular pupil and rotationally symmetric piecewise quadratic diffractive structure. Two example applications to bifocal and trifocal diffractive lenses have been illustrated. This technique can be further applied to binary and N-step diffractive lenses, as well as cases the annular zone area is non-uniform. Extension of this technique to the cases of non-rotational symmetry can also be performed by extending the definition of diffraction efficiency to additional low order terms in the PSF expansion of Eq. 10.

\section{REFERENCES}

[1] "Lenses: Multi-layer Diffractive Optical Element" http://cpn.canon-europe.com/content/education/infobank/lenses/multi layer_diffractive optical element.do

[2] Davison, J. A., Simpson, M. J., "History and development of the apodized diffractive intraocular lens," J. Cataract Ref. Surg. 32, 849-858 (2006).

[3] Lee, S., Choi, M., Xu, Z., Zhao, Z., Alexander E., Liu, Y., "Optical bench performance of a novel trifocal intraocular lens compared with a multifocal intraocular lens," Clin. Ophthalmol. 10, 1031-1038 (2016).

[4] A. J. E. M. Janssen, "New analytic results for the Zernike circle polynomials from a basic result in the Nijboer-Zernike diffraction theory,” J. Eur. Opt. Soc. 6, 11028 (2011). 